\title{
Optimal Power Flow Solution for Wind Integrated Power in Presence of VSC-HVDC Using Ant Lion Optimization
}

\author{
Ramzi Kouadri ${ }^{1}$, Linda Slimani ${ }^{2}$, Tarek Bouktir ${ }^{3}$, Ismail Musirin ${ }^{4}$ \\ ${ }^{1,2,3}$ Faculty of Electrical Engineering, University of Ferhat Abbas Setif 1, Algeria \\ ${ }^{4}$ Faculty of Electrical Engineering, Universiti Teknologi MARA (UiTM), 40450 Shah Alam, Selangor, Malaysia
}

\section{Article Info \\ Article history: \\ Received Apr 29, 2018 \\ Revised Jun 29, 2018 \\ Accepted Jul 11, 2018}

\section{Keywords:}

Ant Lion Optimize (ALO)

Optimal Power Flow (OPF)

Total production cost (TPC)

Voltage Source Converter

Based High Voltage DC (VSC-

HVDC)

Wind Power Generation (WPG)

\begin{abstract}
This paper studies the impact of incorporating wind power generation WPG on the power system on prsence of voltage source converter based high voltage DC (VSC-HVDC). A new meta-heuristic optimization technique are use for solving of the optimal power flow (OPF) problem, this technique optimization namely Ant Lion Optimizer (ALO). The optimization method is the Ant Lion Optimizer (ALO) method for resolve the optimal power flow $(\mathrm{OPF})$ with incorporating of wind power generation on prsence of VSCHVDC. And we used weibull distribution model of the wind farm. The ALOOPF method has been examined and tested on standard test systems IEEE 30 bus with objective functions is minimization of cost total of production TPC are contain the sum of thermal and wind generation cost.
\end{abstract}

Copyright $(0) 2018$ Institute of Advanced Engineering and Science. All rights reserved.

\section{Corresponding Author:}

Ramzi Kouadri,

Faculty of Electrical Engineering,

University of Ferhat Abbas Setif 1, Algeria.

Email: ramzikouadri@gmail.com

\section{INTRODUCTION}

In recent years, consumption of electric power has increased significantly, so it is necessary to find a solution to this increasing demand. Therefore, the incorporating of renewable energy as wind energy into the energy system is one of the best solutions to solve this problem. This source is considered one of the desirable renewable energy sources because it is not polluted and not expensive. Integrating wind farms in presence of High Voltage Direct Current (HVDC) to power grid will inevitably present a big impact on the electrical power system safety and economical operation of the system and such impact has become a lot research topic as wind farm power penetrations increase in power system in recent years [1]. The VSCHVDC transmission is gaining a lot of interest in the power industry, and are much more preferable than AC ones for high voltage levels because they are more economic and more reliable, transmission capacity is greater in HVDC lines due to the non-existence of the skin effect, and also the efficiency and controllability of DC converters are higher [2]. In this scenario, it necessary to solve the optimal power flow (OPF) problem by using Meta heuristic algorithms when the wind power generation (WPG) are introduced in electrical system [3] in presence of VSC-HVDC, where the objective is to find the best operation of electrical power system which minimizes the total production cost and reduction of power losses for a best balance of electrical power system.

Numerous Meta heuristic algorithms are used for solving OPF problem. Several methods are implemented on optimal power flow problem such as, differential algorithm (DE) [4,5], Grey wolf optimizer (GWO) [5], moth-flame optimizer (MFO) [6], moth swarm algorithm (MSA) [7],artificial bee colony algorithm (ABC) [8] and particle swarm optimization (PSO) [9], The Ant Lion Optimization (ALO) technique is one of the modern meta-heuristic algorithms for solving the optimization problem, more 
research applied this algorithm for solving the optimization problem such as in[10], the authors applied ALO algorithm for solving optimal power flow problem with objective function is minimization of total fuel cost, total gas emission and total active losses. Also in [11], the authors applied ALO for objective functions are minimization of Fuel Cost, Voltage Deviation, and improvement of Voltage Stability.

Nowadays, the optimal power flow for the system that includes wind farm power is the subject of ongoing research model. In this paper optimal power flow is carried out for combined wind power farm to electrical power system with and without in presence of VSC-HVDC by using the ant lion optimization (ALO) technique and using weibull distribution model of the wind farm, is best suited probability density function for modelling the wind farm output [12]. Panda and Tripathy in [13] applied modified bacteria foraging algorithm (MBFA) for solving optimal power flow of wind integrated power system with an objective functions is minimization of total cost production (thermal and wind generation) and in[14] applied MBFA for solving security-constrained optimal power flow solution of wind-thermal generation system. In [15], the authors applied three powerful meta-heuristic algorithms-namely, the cuckoo search algorithm (CSA), firefly algorithm (FFA), and flower pollination algorithm (FPA) to optimize power flow in Algeria's Adrar power with Multi-objective function includes: the fuel cost, the NOx emissions, and the imbalance cost of the WPGs. Kiran et al [16] applied Multi-objective hybrid PSO-APO algorithm to solve the multiobjective security constrained optimal power flow (MO-SCOPF) problem.

The objective of this paper is to optimize an objective function to solve constraint based quadratic cost function. Ant Lion Optimization is employed for solving an optimal power flow problem includes wind power in presence of VSC-HVDC. The proposed algorithm is applied to minimization of cost total of production contain the thermal and wind generation cost. The proposed algorithm is tested on IEEE 30-bus, and results of simulation are compared with two methods WOA and GWO methods.

\section{MODEL OF VSC-HVDC}

This section introduces the modeling of the AC/HVDC system. The modeling is based in the converter station and DC grids system models as shown in Figure 1. The model a converter station consists of a controllable voltage source $\underline{U}_{c}=U_{c} \angle \delta_{c}$ behind the phase reactor, represented as complex impedance $\underline{Z}_{c}=R_{c}+j X_{c}$. the low pass filter from Figure 2 is represented as a susceptance $B_{f}$ at system frequencies. A transformer connects the filter bus to the AC grid and is represented by its complex impedance $\underline{Z}_{t f}=R_{t f}+$ $j X_{t f}[17]$.

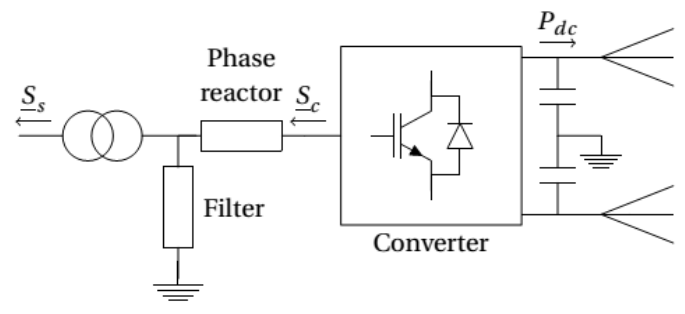

Figure 1. VSC MTDC converter station [17]

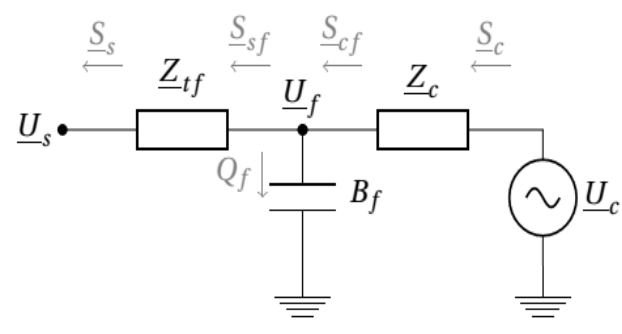

Figure 2. Equivalent single phase power flow model

The equations for active and reactive power at the grid side in terms of the complex voltages are:

$$
\begin{aligned}
& P_{S}=-U_{S}^{2} G_{t f}+U_{s} U_{f}\left[G_{t f} \cos \left(\delta_{s}-\delta_{f}\right)+B_{t f} \sin \left(\delta_{s}-\delta_{f}\right)\right] \\
& Q_{s}=U_{s}^{2} B_{t f}+B_{s} B_{f}\left[G_{t f} \sin \left(\delta_{s}-\delta_{f}\right)-B_{t f} \cos \left(\delta_{s}-\delta_{f}\right)\right]
\end{aligned}
$$

The equations at the converter side are:

$$
\begin{aligned}
& P_{c}=U_{c}^{2} G_{c}-U_{f} U_{c}\left[G_{c} \cos \left(\delta_{f}-\delta_{c}\right)-B_{c} \sin \left(\delta_{f}-\delta_{c}\right)\right] \\
& Q_{c}=-U_{c}^{2} B_{c}+U_{f} U_{c}\left[G_{c} \sin \left(\delta_{f}-\delta_{c}\right)+B_{c} \cos \left(\delta_{f}-\delta_{c}\right)\right]
\end{aligned}
$$


The expressions for the filter side complex power flowing through the transformer are written as

$$
\begin{aligned}
& P_{s f}=U_{f}^{2} G_{t f}-U_{f} U_{s}\left[G_{t f} \cos \left(\delta_{s}-\delta_{f}\right)-B_{t f} \sin \left(\delta_{s}-\delta_{f}\right)\right] \\
& Q_{s f}=-U_{f}^{2} B_{t f}+U_{f} U_{s}\left[G_{t f} \sin \left(\delta_{s}-\delta_{f}\right)+B_{t f} \cos \left(\delta_{s}-\delta_{f}\right)\right]
\end{aligned}
$$

And those flowing through the phase reactor side are

$$
\begin{aligned}
& P_{c f}=-U_{f}^{2} G_{c}+U_{f} U_{c}\left[G_{c} \cos \left(\delta_{f}-\delta_{c}\right)+B_{c} \sin \left(\delta_{f}-\delta_{c}\right)\right] \\
& Q_{c f}=U_{f}^{2} B_{c}+U_{f} U_{c}\left[G_{c} \sin \left(\delta_{f}-\delta_{c}\right)-B_{c} \cos \left(\delta_{f}-\delta_{c}\right)\right]
\end{aligned}
$$

The DC system modeling can be represented by a resistive network with current injections and DC voltages at the different nodes[18]. The current injected at a DC node $\mathrm{i}$ and the power flow equation of DC line can be written as:

$$
\begin{aligned}
& I_{d c_{i}}=Y_{d c_{i j}} \cdot\left(U_{d c_{i}}-U_{d c_{j}}\right) \\
& P_{d c_{i}}=p U_{d c_{i}} Y_{d c_{i j}} \cdot\left(U_{d c_{i}}-U_{d c_{j}}\right)
\end{aligned}
$$

where $Y_{d c_{i j}}$ equal to $1 / R_{d c_{i j}}$ and $p=1$ for a monopolair system or $p=2$ for a bipolair system.

\section{FORMULATION PROBLEM}

\subsection{Problem}

The objective of the OPF problem is to minimize the total cost of all available generators plants subjected to the operating constraints (equality and inequality constraints) in the power system. In this paper, the objective function is the sum of the operating costs of each available conventional generator and the wind farms can be expressed as follow:

$$
F=\sum_{i=1}^{T P G} C_{t}\left(P_{G i}\right)+\sum \gamma_{p . w . j}\left(P w_{j . a v}-P w_{j}\right)+\sum \operatorname{lm}_{r . w . j}\left(P w_{j}-P w_{j . a v}\right)
$$

In this expression. The first term denote the cost of thermal power generation, second term is the cost due to under estimation of available wind power and third term is the cost due to over estimation of available wind power, Details of the first, second and third terms are explained as:

\subsection{Fuel cost of the conventional generator}

Widely acclaimed quadratic cost function for the thermal generators are mathematically represented by (12) as follows:

$$
C_{t}\left(P_{G i}\right)=A_{i}+B_{i} P_{G i}+C_{i} P_{G i}^{2}
$$

The cost of wind power generation (WPG) is calculated considering the over-generation and undergeneration of the available wind power, based on the Weibull distribution function WDF of the wind speed [19], represented by the second and third terms of Equation (11).

\subsection{Cost function due to the over-generation}

The cost function due to over generation of available wind power is expressed as follow [19]:

$$
C_{p . W . i}\left(W_{i . a v}-W_{i}\right)=k_{p . i}\left(W_{i . a v}-W_{i}\right)=k_{p . i} \int_{W_{i}}^{W_{r . i}}\left(W-W_{i}\right) f_{W}(W)
$$

Where $k_{p . i}$ is the penalty cost coefficient for over-generation of $i^{t h}$ wind farm, $f_{W}(W)$ is the probability density function (PDF) of wind power output.

\subsection{Cost function due to the under-generation}

The cost function of $i^{t h}$ wind farm for calling the reserves to cover $i^{t h}$ wind farm due to undergeneration is expressed as follows [20, 21]: 


$$
C_{r . W . i}\left(W_{i}-W_{i . a v}\right)=k_{r . i}\left(W_{i}-W_{i . a v}\right)=k_{r . i} \int_{W_{i}}^{W_{r . i}}\left(W-W_{i}\right) f_{W}(W)
$$

Where $k_{r . i}$ is the reserve cost coefficient for under-generation of $i^{\text {th }}$ wind farm.

The probability density function (PDF) of the Wiebull distribution is given by:

$$
f_{V}(V)=\frac{k}{c}\left(\frac{v}{c}\right)^{k-1} e^{\left[\left(-\frac{v}{c}\right)^{k}\right]}
$$

Where $\mathrm{v}$ is the wind speed, $\mathrm{k}$ and $\mathrm{c}$ are the shape factor and scale parameter $(\mathrm{m} / \mathrm{s})$.

For the Weibull distribution function, the discrete portions of wind energy conversation system (WECS) power output random variable as following [19]:

$$
\begin{aligned}
& P_{\text {rated }}\{W=0\}=1-\exp \left(-\left(\frac{V_{\text {cut-in }}}{c}\right)^{k}\right)+\exp \left(-\left(\left(\frac{V_{\text {cut }- \text { off }}}{c}\right)^{k}\right)\right) \\
& P_{\text {rated }}\left\{W=W_{\text {rated }}\right\}=\exp \left(-\left(\frac{V_{\text {rated }}}{c}\right)^{k}\right)+\exp \left(-\left(\left(\frac{V_{\text {cut }- \text { off } f}}{c}\right)^{k}\right)\right)
\end{aligned}
$$

While the continuous portion of WECS power output random variable in becomes:

$$
f_{W}(W)=\frac{k l v_{c u t-i n}}{c}\left(\frac{(1+\rho * 1) v_{c u t-i n}}{c}\right)^{k-1} \exp \left(-\left(\frac{(1+\rho * 1) v_{c u t-i n}}{c}\right)^{k}\right)
$$

\section{THE ANT LION OPTIMIZATION ALGPRITHM (ALO)}

Ant lion optimization algorithm ALO is a new nature inspired proposed by Seyedali Mirjalili in 2015 [22], for to solve any optimization problems of constrained engineering. The ALO algorithm modelled based on hunting mechanism of antlions in nature. The benefits of ALO algorithm, it can find optimal solution for minimizing the objective function. In ALO algorithm five main steps of hunting prey as the random walk of ants, trapping in antlions traps, building traps, sliding ants against toward antlion, Catching preys and rebuilding traps are implemented.

The ALO algorithm mimics the hunting behavior of ant lions, i.e., the interaction between predator (ant lions) and prey (ant).

In this algorithm, the expression mathematically of is the random walks of ants for detects the location of food is describes in equation (19).

$$
X(t)=\left[0, \operatorname{cumsu}\left(2 r\left(t_{1}\right)-1\right), \operatorname{cumsu}\left(2 r\left(t_{2}\right)-1\right), \ldots, \operatorname{cumsu}\left(2 r\left(t_{n}\right)-1\right)\right]
$$

where $X(t)$ is the random walks of ants, $n$ is the max-iterations, $t$ is the step of random walk, and $r(t)$ is a function defined as follows:

$$
r(t)=\left\{\begin{array}{l}
1 \text { if rand }>0.5 \\
0 \text { if rand }<0.5
\end{array}\right.
$$

where, rand is a randomly generated number uniformly distributed in the range of $[0,1]$.

The Details of different steps of ALO algorithm describe the relation between predators (ant lions) and prey (ant) are explained as.

\subsection{Random walk of ants}

In every step of optimization, ants update their positions with random walk and Random walks are all based on the Equation (21), all the positions of ants are inside the boundary of the search space, since every search space has a boundary, the random walks of ants is normalized by using the following equation:

$$
X_{i}^{t}=\frac{\left(X_{i}^{t}-a_{i}\right) *\left(b_{i}^{t}-c_{i}^{t}\right)}{\left(b_{i}-a_{i}\right)}+c_{i}^{t}
$$


where the $a_{i} ; b_{i}$ are respectively the minimum and maximum of random walk corresponding of $i^{\text {th }}$ variable. $c_{i}^{t} ; d_{i}^{t}$ : are respectively indicated the minimum and maximum of $i t h$ variables at $t$ th iteration.

\subsection{Trapping in antlions traps}

The influence of antlions traps on random walks of ants is expressed as follow:

$$
\begin{aligned}
& c_{i}^{t}=\text { Antlion }_{j}^{t}+c^{t} \\
& d_{i}^{t}=\text { Antlion }_{j}^{t}+d^{t}
\end{aligned}
$$

\subsection{Building traps}

During optimization, The ALO algorithm is required to use a roulette wheel operator for selecting antlions based of their fitness for give a high chance for catching ants.

\subsection{Sliding ants against toward antlion}

With the mechanisms proposed so far, antlions are capable to build traps proportional to their fitness and ants are required to move randomly near of the center of pit. However, once antlions realize that an ant is in the trap, they shoot sands outwards the center of the pit. This mechanism mathematically is proposed modeled as follow:

$$
\begin{aligned}
& c^{t}=\frac{c^{t}}{I} \\
& d^{t}=\frac{d^{t}}{I}
\end{aligned}
$$

\subsection{Catching preys and rebuilding the traps}

The final step of hunting is when an ant reaches in the bottom of the antlion pit and is caught in the antlion's jaw. After this step, the predator pulls the ant inside the sand and consumes its body. Then the antlion is required to update its position to the latest position of the hunted ant to enhance its chance of catching new prey. Catching prey and re-building the pits calculated as given by the following equation:

$$
\text { Antlion }_{i}^{t}=\text { Ant }_{i}^{t}, \text { if } f\left(\text { Ant }_{i}^{t}\right)>f\left(\text { Antlion }_{j}^{t}\right)
$$

where Antlion $_{i}^{t}$, Ant $_{i}^{t}$ is jth the position of the selected antlion and ant at iteration $t$.

\subsection{Elitism}

Elitism is one of the most important characteristic of all evolutionary algorithms. Elitism of ant lion is calculated by using the roulette wheel. In this study, the best solution(s) obtained by the ALO algorithm at any iteration is saved as elite. The elitism mechanism mathematically described as follows equation:

$$
A n t_{i}^{t}=\frac{R_{A}^{t}+R_{E}^{t}}{2}
$$

Where $R_{A}^{t} \mathrm{~A}$ is the random walk around the antlion and is selected by using the roulette wheel at th iteration, $R_{E}^{t} \mathrm{E}$ is the random walk around the elite at th iteration

\section{RESULTS AND ANALYSIS}

In this study for the purpose of verifying the performance and efficiency of the ALO algorithm proposed on the power system with and without incorporating wind power generation and presence of VSCHVDC, tests are carried out on IEEE 30 bus system.. All the simulations are carried out by using MATLAB 2009b, and computed Core (TM i5; CPU $1.80 \mathrm{GHz}-8 \mathrm{Go}$ RAM). For establishing the superiority of the proposed ALO, 30 independent trial runs are performed for all the test cases with a comparative study reported in the following section, the population size $(\mathrm{NP}=40)$ and number of iterations maximal (iter Max $=500)$ are taken same for three optimization methods ALO, GWO and WOA.

\subsection{Results of IEEE 30-bus test power system without WPG and VSC-HVDC}

To verify the proposed approach and for comparison purposes, we perform simulations on the IEEE 30-bus system, involves 6 generators, 41 lines, 4 transformers. About this case study, 9 reactive 
compensators are installed at buses. The load demand is 283.4 MW and 73.5 MVar n 100 MVA base. The results presented in Table 1 including the generation cost and real power losses of the proposed method and with other existing optimization methods.

Table 1. Comparison of Fuel Cost Obtained By Proposed Algorithm and Other Algorithms.

\begin{tabular}{|c|c|c|c|c|c|c|c|c|}
\hline Variables & $\min \max$ & ALO & GWO & WOA & $\mathrm{DE}(4)$ & GWO(5) & MFO(6) & $\operatorname{MSA}(7)$ \\
\hline$P_{\text {loss }}(\mathrm{MW})$ & & 8.6026 & 8.8347 & 8.7405 & 8.6150 & 9.30 & 9.3000 & 9.0345 \\
\hline Cost $(\$ / h r)$ & & 799.0133 & 799.2954 & 799.8095 & 799.2891 & 801.41 & 799.072 & 800.5099 \\
\hline
\end{tabular}

In order to verify and test the robustness and performs of the proposed algorithm for optimal solution of minimizing the objective function, we made a comparison between of the proposed method with other existing optimization methods presented in the literature. Here, we considered fuel cost of thermal generators as an objective function. The comparison of minimum fuel cost and minimum power losses obtained by the proposed method (ALO) with other existing methods as GWO, WOA, DE, GWO, MFO, and MSA has been presented in Table 1. From the results, the minimum fuel cost and minimum power active losses obtained by the proposed ALO method are $799.0133 \$ / \mathrm{h}$ and $8.6026 \mathrm{MW}$ respectively, which are better than all other algorithms as well as previously reported methods. The proposed ALO optimization method superior and robust compared to GWO and WOA algorithms in terms of getting near global optimum point and faster convergence for solving the OPF problem. The convergence characteristics of the proposed method and, GWO and WOA methods are shown in Figure 3.

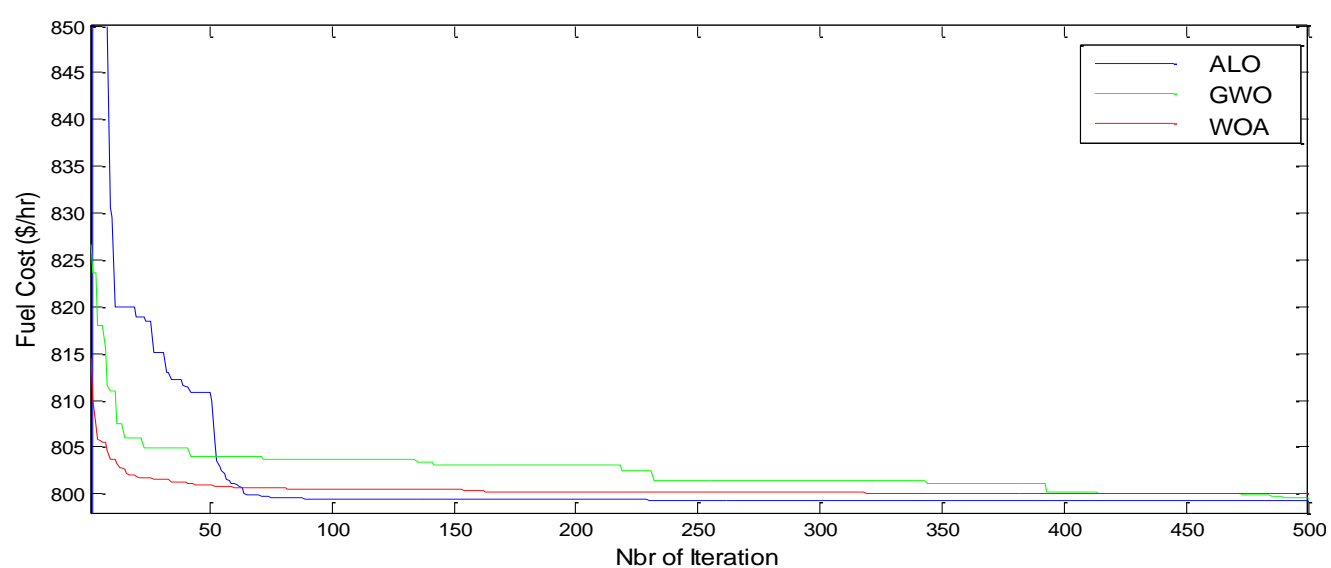

Figure 3. IEEE 30-bus system: Convergence plot for ALO, GWO and WOA methods

\subsection{Results of IEEE 30-bus with WPG and VSC-HVDC}

In this cases, we have study the influence of wind power generation WPG on power system in presence of VSC-HVDC device as minimization of total production cost (TPC) and reduction of power losses, The proposed ALO algorithm is tested by using Weibull distribution model of the wind farm with objective function is minimization of thermal generation cost (TGC) and wind generation cost (WGC), and its performance is compared with the standard GWO and WOA algorithms. To verify the performance and effectiveness of the proposed algorithm of solving OPF problem including wind power farm with and without presence of VSC-HVDC, two different case studies are considered, the case 1 in this studies is the base case (including wind power farm without VSC-HVDC), the second case is including wind power farm with presence in VSC-HVDC. Two wind farm (WF) consists of 43 units of wind turbine generation (WTG) are connected to the system at bus no 10 and bus 24 , a nominal power rating of each WTG is 2 MW with a total capacity are $85.02 \mathrm{MW}$, the wind power generation of WF1 at bus No. 10 having a total capacity of $56.68 \mathrm{MW}$ and the wind power generation of WF2 consists a total capacity of $28.34 \mathrm{MW}$, This two values of wind power was taken from [1] and are considered as the best penetration of wind power.

The parameters of wind turbine are the factors of wind speed distribution are: shape factor, $\mathrm{k}=1.12$ and scale factor, $\mathrm{c}=5.92$, and the characteristics wind turbine on this studies are: $P_{\text {rated }}=2000 \mathrm{KW}$, 
$V_{\text {rated }}=10.28 \mathrm{~m} / \mathrm{s}, V_{\text {cut-in }}=4 \mathrm{~m} / \mathrm{s}, V_{\text {cut-off }}=25 \mathrm{~m} / \mathrm{s}$. The penalty factor is $k_{p . j}=0.03 \$ / M W h$ and the reserve factor is $k_{r . j}=0.03 \$ / M W$.

\section{Case 1: ALO Solution without VSC-HVDC}

The first case studies, the proposed ALO algorithm are tested for case of increased load at $45 \%$ from total load for see the effect of wind energy on the system in case of contingency, the total load was 410.93 MW. The results obtained by the proposed method and with those found by GWO and WOA are presented in the Table 2.

Table 2. Comparison of fuel cost obtained by proposed algorithm and other reported algorithms case of

\begin{tabular}{ccccc}
\multicolumn{5}{c}{ contingency } \\
\hline Variables & Without WPG & ALO & GWO & WOA \\
\hline$P_{\text {loss }}(\mathrm{MW})$ & 13.7979 & 11.4941 & 11.7777 & 11.7524 \\
Thermal generation cost $(\$ / h r)$ & 1339.6 & 970.8575 & 971.1843 & 977.8275 \\
Wind generation cost $(\$ / h r)$ & $/ / I$ & 19.9495 & 19.9529 & 19.9515 \\
Total production cost $(\$ / h r)$ & 1339.6 & 990.8070 & 991.1372 & 997.7790 \\
\hline
\end{tabular}

The Table 2 shows, real power losses and total production cost (TPC) by the proposed method and two optimization methods in literature are GWO and WOA. The active power loss was reduced from the value of 13.7979 MW (without WPG) to $11.5420 \mathrm{MW}$ in case of integration of WPG. Therefore, it is clear to see effect of wind power generation (WPG) on the power system in case of contingency. The results obtained by ALO algorithm shown better results for the real power losses and the total cost of production in the values 11.5420 MW and 990.0337 \$/hr respectively compared results by GWO and WOA.

\section{Case 2: ALO Solution for over-load with WPG and VSC-HVDC}

In this part, we have use VSC-HVDC device for a best soultion in problem of over-load, transprted maximum energy and reduction of active power losses. In this case study, a VSC-HVDC is integrated in the IEEE 30 bus system between bus 2 and 5. The DC system voltage is $+/-132 \mathrm{kV}$. The converter station VSC1 (slack bus of the DC network) is connected to bus 2 and VSC2 is connected to bus 5 of the AC system. The parameters and at the converter stations are given in Table 3 and the results obtained by the proposed method are presented in the Table 4.

Table 3. VSC Converter Data

\begin{tabular}{cccc}
\hline \multirow{2}{*}{ Converter parameters } & \multicolumn{2}{c}{ Rating \& Converter loss data } \\
\cline { 2 - 4 } & & No. & 1,2 \\
\hline$X_{t r}(p . u)$ & 0.1121 & $\pm V_{d c}$ & 132 \\
$R_{t r}(p . u)$ & 0.0015 & $P_{d c}(M W)$ & 90 \\
$B_{f}(p . u)$ & 0.0887 & $a$ & 1.103 \\
$X_{c}(p . u)$ & 0.1642 & $b$ & 0.887 \\
$R_{c}(p . u)$ & 0.0001 & $c_{\text {rec }}$ & 2.885 \\
$R_{d c 2-5}(p . u)$ & 0.0472 & $c_{\text {inv }}$ & 4.371 \\
\hline
\end{tabular}

Table 4. Comparison of results obtained by proposed algorithm with and without VSC-HVDC

\begin{tabular}{cccc}
\hline Variables & Without WPG and HVDC & With WPG & With WF and HVDC \\
\hline$P_{\text {loss }}(\mathrm{MW})$ & 13.7979 & 11.4941 & 11.0183 \\
Thermal generation cost $(\$ / h r)$ & 1339.6 & 971.1843 & 970.1096 \\
Wind generation cost $(\$ / h r)$ & 19.9495 & 19.9529 & 19.9529 \\
Total production cost $\$ / h r)$ & 1339.6 & 991.1372 & 990.0625 \\
\hline
\end{tabular}

from Table 4 , the presence in HVDC line between buses 2 and 5 reduce the total production cost, also the active power losses was reduced from the value of 11.4941MW (without HVDC line) to 11.0183 [MW] in presence of HVDC line. Also from Figure 4, after the increase in the load at $45 \%$ (case without presence in HVDC line ), the power transmitted in line 5 between bus 2 and 5 is $80.00 \mathrm{MW}$ (this value is the maximum limit $80 \mathrm{MW}$ ) and the power transmitted in line 1 between bus 1 and 2 is $127.7722 \mathrm{MW}$ (this value 
is near the maximum limit $130 \mathrm{MW}$ ), after integration of HVDC in line 5 (line 5: is optimal placement of HVDC device in IEEE 30 bus), the power transmitted in line 1 is decreased to equal $125.33 \mathrm{MW}$, the power transmited in HVDC line is $90 \mathrm{MW}$, and see that also the effect of VSC-HVDC to find a best solution for the problem of over-load are shown in Figure 4 by using the proposed method.

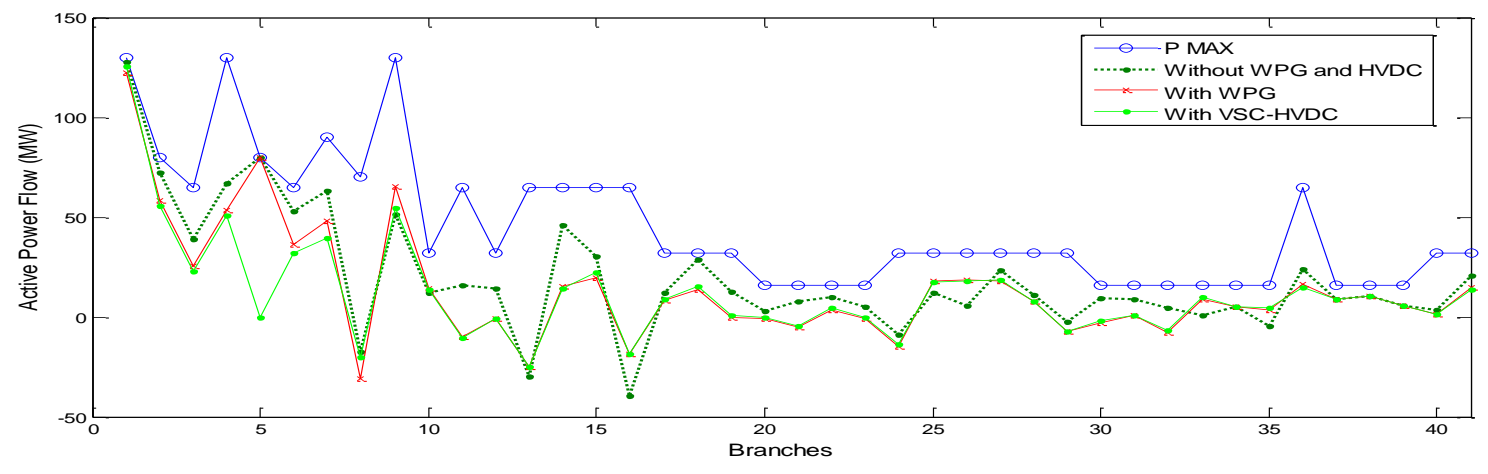

Figure 4. The effect of WPG on the Active powers transit with and without HVDC Line

\section{CONCLUSION}

In this paper, a recent metaheuristic technique namely Ant Lion Optimizer (ALO) was successfully applied to solve the optimal power flow (OPF) problem for case of integration of power wind farm on the power system with and without in presence of VSC-HVDC. We studied an optimal power flow for minimization of total production cost. The proposed ALO method has been examined and tested on the IEEE 30-bus. The obtained results of ALO method were compared with those obtained from two methods developed by us, namely, Grey wolf Optimization and the Whole Optimizer Algorithm. The Ant Lion Optimizer (ALO) method has been a best result. The Simulation shows the best results for minimize the cost of generation, the active power losses and reduction of over-load in the transmission line when the WPG includes in the power system in presence of VSC-HVDC.

\section{REFERENCES}

[1] L. Slimani, T Bouktir. Application of Differential Evolution Algorithm to Optimal Power Flow with High Wind Energy Penetration. Acta Electrotehnica. 2012; 53(1): 59-68.

[2] M. J.Carrizosa, F. D.Navas, G. Damm. Optimal power flow in multi-terminal HVDC grids with offshore wind farms and storage devices. Electrical Power and Energy Systems. $2015 ; 65: 291-298$.

[3] S. Li, D. C.Wunsch, E. A. O"Hair, and M. G. Giesselmann. Using neural networks to estimate wind turbine power generation. IEEE Trans. Energy Convers. 2001; 16(3): 276-282.

[4] A.A. Abou El Ela, M.A. Abido and S.R. Spea. Optimal power flow using differential evolution algorithm. Electric Power Systems Research. 2010; 80: 878-885.

[5] A. A. El-Fergany and H. M. Hasanien. Single and Multi-objective Optimal Power Flow Using Grey Wolf Optimizer and Differential Evolution Algorithms. Electric Power Components and Systems, 2015; 43(13): 1548-1559.

[6] I. N. Trivedi, P. Jangir, S. A. Parmar, N. Jangir. Optimal power flow with voltage stability improvement and loss reduction in power system using Moth-Flame Optimizer. Neural Computing and Applications, Dec 2016.

[7] A.-A.A. Mohamed, Y. S. Mohamed and A. A.M. El-Gaafary. Optimal power flow using moth swarm algorithm. Electric Power Systems Research. 2017; 142: 190-206.

[8] M. R. Adaryani and A. Karami. Artificial bee colony algorithm for solving multi-objective optimal power flow problem. Electrical Power and Energy Systems. 2013; 53: 219-230.

[9] M.A. Abido. Optimal power flow using particle swarm optimization. Int $j$ Electr Power Energy Syst 2002, 24(7):563-71.

[10] S. Ahmed, N. Djemai and T. Bouktir. Optimal Power Flow Solution using Ant Lion Optimizer Algorithm. International Conference on Recent Advances in Electrical Systems. 2016: 13-18.

[11] I. N. Trivedi, S. A. Parmar, R. H. Bhesdadiya and P. Jangir, "Voltage Stability Enhancement and Voltage Deviation Minimization Using Ant-Lion Optimizer Algorithm", International Conference on Advances in Electrical, Electronics, Information, Communication and Bio-Informatics, 2016.

[12] S. Velamuri, S. Sreejith, P. Ponnambalam. Static economic dispatch incorporating wind farm using Flower pollination algorithm. Perspectives in Science. 2016; 8: 260-262.

[13] A. Panda, M. Tripathy. Optimal power flow solution of wind integrated power system using modified bacteria foraging algorithm. Electrical Power and Energy Systems. 2014; 54: 306-314. 
[14] A. Panda and M. Tripathy. Security constrained optimal power flow solution of wind-thermal generation system using modified bacteria foraging algorithm. Energy. 2015; 93: 816-827.

[15] S. Makhloufi, A. Mekhaldi, and M. Teguar. a Three powerful nature-inspired algorithms to optimize power flow in Algeria's Adrar power system. Energy. 2016.

[16] K. Teeparthi and D.M. V. Kumar, "Multi-objective hybrid PSO-APO algorithm based security constrained optimal power flow with wind and thermal generators", Engineering Science and Technology, an International Journal. 2017; 20: 411-426.

[17] Beerten, J., Cole, S., Belmans, R. "Generalized steady-state VSC MTDC model for sequential AC/DC power flow algorithms", IEEE Trans. Power Syst. 2012; 27: 821-829.

[18] J. Beerten. MATACDC User's Manual. [Online].Avail able:http://www.esat.kuleuven.be/electa/teaching/matacdc/MatACDCManual, 2012.

[19] Hetzer J, Yu DC and Bhattarai K. An economic dispatch model incorporating wind power. IEEE Trans Energy Convers. 2008; 23(2): 603-611.

[20] L.B. Shi, C. Wang, L.Z. Yao, L.M. Wang, Y.X. Ni and B. Masoud. Optimal Power Flow with Consideration of Wind Generation Cost. International Conference on Power System Technology (POWERCON), IEEE Conference Publications. 2010: 1- 5.

[21] R.A. Jabr and B.C. Pal. Intermittent Wind Generation in Optimal Power Flow Dispatching. Generation, Transmission and Distribution, IET, Journals \& Magazines. 2009; 3(1): 66 -74.

[22] S. Mirjalili. The ant lion optimizer. Adv. Eng. Softw. 2015; 83: 80-98. 